\title{
Adenoma of the nonpigmented ciliary epithelium presenting with recurrent iridocyclitis: unique expression of glial fibrillary acidic protein
}

\author{
Jeong Hun Bae • Ji Eun Kwon • Woo Ik Yang • \\ Sung Chul Lee
}

Received: 17 September 2010 /Revised: 18 October 2010 / Accepted: 21 October 2010 /Published online: 19 November 2010

(C) The Author(s) 2010. This article is published with open access at Springerlink.com

Dear Editor,

Adenoma of the nonpigmented ciliary epithelium (NPCE) is extremely rare and may mimic malignant melanoma. It has variable clinical and histopathological features. Here, we provide the first report of a case of an adenoma of the NPCE that presents with recurrent iridocyclitis, showing positive immunostaining for glial fibrillary acidic protein (GFAP) in the tumor.

An 18-year-old boy was referred for a nonpigmented iris mass in his right eye. Before the detection of this mass, he had been treated for recurrent iridocyclitis for 2 years. Bestcorrected visual acuity (BCVA) was 20/20, and intraocular pressure was $17 \mathrm{mmHg}$ in the right eye. Slitlamp biomicroscopy revealed $2+$ anterior chamber cells and a

Conflict of interest The authors have no proprietary interest.

Financial Disclosure None

Patient Consent Informed consent was obtained for publication of figures.

J. H. Bae

Department of Ophthalmology, Kangbuk Samsung Hospital,

Sungkyunkwan University School of Medicine,

Seoul, Korea

J. E. Kwon • W. I. Yang

Department of Pathology, Yonsei University College of Medicine,

Seoul, Korea

S. C. Lee $(\bowtie)$

Department of Ophthalmology,

Yonsei University College of Medicine,

134 Shinchon-dong, Seodaemun-gu,

Seoul 120-752, Korea

e-mail: sunglee@yuhs.ac grayish-white mass with a well-demarcated margin and prominent vascularization at the 5 o'clock position (Fig. 1a). Gonioscopy showed that the mass, which arose from the ciliary body, partially covered the iris surface and the anterior chamber angle. Ultrasound biomicroscopy revealed medium internal reflectivity in the mass, measuring $3.0 \times 2.3 \mathrm{~mm}$ (Fig. 1b). The differential diagnosis included uveal melanoma, ciliary epithelial tumor, ciliary body granuloma, and leiomyoma. Partial lamellar sclerouvectomy was performed without complications. One year after surgery, BCVA was 20/20, and there was no evidence of recurrence of the tumor or intraocular inflammation.

Microscopically, the tumor consisted of sheets, cords, and tubules occasionally forming glandular configurations (Fig. 1c). The tumor cells had abundant eosinophilic cytoplasm with bland-looking round nuclei and moderately prominent nucleoli. The cellular borders were scalloped due to cytoplasmic indentation and artifactural clefting. The tumor cell nests were surrounded by abundant hyalinized stroma. Neither necrosis nor mitotic figures were observed. Immunohistochemical analyses were positive for S-100 protein, cytokeratins (AE1/AE3), vimentin, and GFAP (Fig. 1d), but negative for HMB-45 and melan A. The histopathological and immunohistochemical findings confirmed the diagnosis of an adenoma of the NPCE.

Adenoma of the NPCE may be congenital or acquired, and shows a wide spectrum of biological behavior [1, 2]. Despite its benign nature, adenoma of the NPCE may have local aggressiveness that results in cataract, glaucoma, vitreous hemorrhage, and intraocular inflammation; therefore, it is 

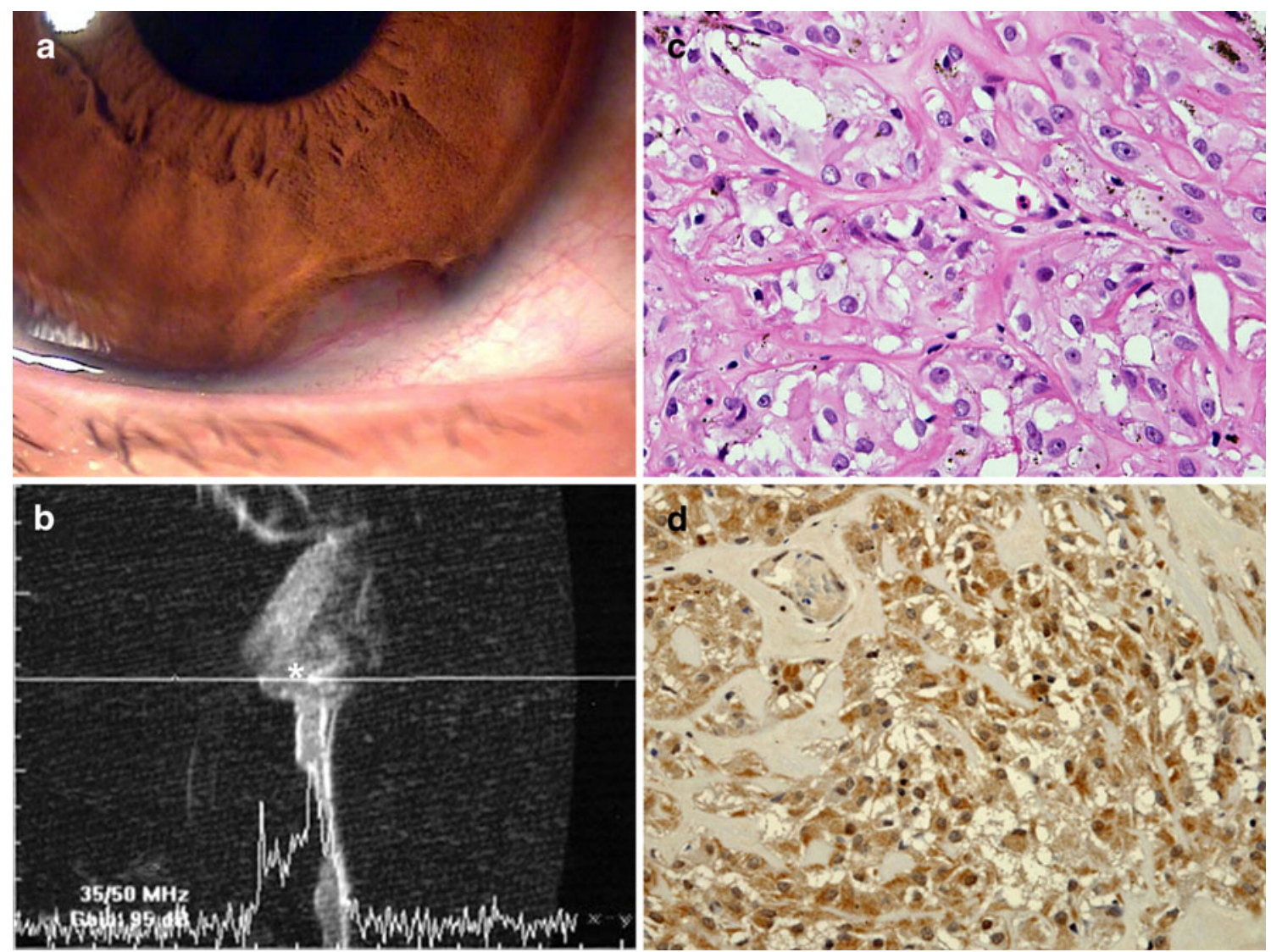

Fig. 1 Slit-lamp photography of a vascularized ciliary body mass extending into the anterior chamber. There is mild conjunctival hyperemia with $2+$ anterior chamber cells (a). Ultrasound biomicroscopy discloses the ciliary body mass $\left(^{*}\right)$ encroaching on the iris and the anterior chamber angle. The A-scan mode demonstrates medium internal reflectivity in the mass (b). The tumor consists of sheets,

difficult to clinically differentiate from malignant tumors of the ciliary body [2]. Our case was diagnosed as an adenoma of the NPCE because of the histopathological features of the tumor and the immunohistochemical stains, which confirmed that the tumor originated from the NPCE $[1,3]$.

Positive immunostaining for GFAP in adenoma of the NPCE, to our knowledge, has never been reported. GFAP is thought to be specific for glial cells, especially astrocytes. In our case, however, astrocytic phenotype was not observed. Recent studies have demonstrated that the adult human ciliary epithelium contains progenitor cells with properties of neural stem cells which express GFAP and have the potential to proliferate and differentiate into both neurons and glia $[4,5]$. Diffuse expression of GFAP may be largely attributed to the neural progenitor cells in the ciliary epithelium. Possible explanations for the intraocular inflammation observed in our case include direct infiltration of the iris by the tumor and altered immune responses of the anterior chamber.

We report a case of an adenoma of the NPCE that presented with recurrent iridocyclitis. Although benign, cords, and tubules forming glandular configurations with abundant hyalinized background. The cells have bland-looking round nuclei and abundant eosinophilic cytoplasm (hematoxylin-eosin, $\times 200)($ c). The tumor cells show moderately positive staining for glial fibrillary acidic protein $(\mathbf{d})$

adenoma of the NPCE may exhibit progressive growth and local invasion, causing intraocular inflammation. Positive expression of GFAP in this tumor has never been reported, but should be considered in the differential diagnosis. Partial lamellar sclerouvectomy seems appropriate to confirm the diagnosis and to preserve a good visual acuity.

Open Access This article is distributed under the terms of the Creative Commons Attribution Noncommercial License which permits any noncommercial use, distribution, and reproduction in any medium, provided the original author(s) and source are credited.

\section{References}

1. Grossniklaus HE, Lim JI (1994) Adenoma of the nonpigmented ciliary epithelium. Retina 14:452-456

2. Mansoor S, Qureshi A (2004) Ciliary body adenoma of nonpigmented epithelium. J Clin Pathol 57:997-998 
3. Shields JA, Eagle RC Jr, Shields CL, De Potter P (1996) Acquired neoplasms of the nonpigmented ciliary epithelium (adenoma and adenocarcinoma). Ophthalmology 103:2007-2016

4. Moe MC, Kolberg RS, Sandberg C, Vik-Mo E, Olstorn H, Varghese M, Langmoen IA, Nicolaissen B (2009) A comparison of epithelial and neural properties in progenitor cells derived from the adult human ciliary body and brain. Exp Eye Res 88:30-38

5. Das AV, Zhao X, James J, Kim M, Cowan KH, Ahmad I (2006) Neural stem cells in the adult ciliary epithelium express GFAP and are regulated by Wnt signaling. Biochem Biophys Res Commun 339:708-716 